\title{
Simulation studies of radiation linewidth in circular Josephson-junction fluxon oscillators
}

\author{
If, F.; Christiansen, Peter Leth; Parmentier, R. D.; Skovgaard, Ove; Sørensen, Mads Peter
}

Published in:

Physical Review B

Link to article, DOI:

10.1103/PhysRevB.32.1512

Publication date:

1985

Document Version

Publisher's PDF, also known as Version of record

Link back to DTU Orbit

Citation (APA):

If, F., Christiansen, P. L., Parmentier, R. D., Skovgaard, O., \& Sørensen, M. P. (1985). Simulation studies of radiation linewidth in circular Josephson-junction fluxon oscillators. Physical Review B, 32(3), 1512-1518. https://doi.org/10.1103/PhysRevB.32.1512

\section{General rights}

Copyright and moral rights for the publications made accessible in the public portal are retained by the authors and/or other copyright owners and it is a condition of accessing publications that users recognise and abide by the legal requirements associated with these rights.

- Users may download and print one copy of any publication from the public portal for the purpose of private study or research.

- You may not further distribute the material or use it for any profit-making activity or commercial gain

- You may freely distribute the URL identifying the publication in the public portal 


\title{
Simulation studies of radiation linewidth in circular Josephson-junction fluxon oscillators
}

\author{
F. If, P. L. Christiansen, R. D. Parmentier, ${ }^{*}$ O. Skovgaard, and M. P. Soerensen \\ Laboratory of Applied Mathematical Physics, The Technical University of Denmark, DK-2800 Lyngby, Denmark
}

(Received 8 November 1984)

\begin{abstract}
Detailed simulation studies of the dynamics of fluxons in long circular Josephson tunnel junctions under the influence of external microwave radiation and internal thermal noise are presented. The simulation algorithm uses a pseudospectral method well adapted to vector processors (CRAY-1-S), which gives a speed-up factor in computing time of typically 22 in comparison to conventional high-speed computers, and also provides results with a relative accuracy of less than $10^{-8}$ thereby making possible the study of the very narrow radiation linewidth of such oscillators. Comparison of calculated linewidths with experimental results shows good agreement.
\end{abstract}

\section{INTRODUCTION}

Josephson-junction fluxon oscillators continue to attract research interest both theoretically, in studies of nonlinear wave dynamics, and experimentally, where the very narrow linewidth of the emitted microwave radiation promises potentially interesting applications. ${ }^{1}$ This very narrow linewidth makes the numerical study of the detailed dynamics of such oscillators very CPU time consuming. In order to overcome these difficulties we have developed a pseudospectral algorithm for solving the perturbed sine-Gordon equation which describes the oscillator. This algorithm employs a Fourier transformation of the spatial variable together with a finite-difference approximation to the time variable. The extensive use of fast Fourier transforms in the algorithm has made the implementation natural on a CRAY-1-S vector processor. The Fourier treatment of the space variable requires spatial periodicity in the model. In physical terms this means that we are studying a circular junction oscillator of the type first proposed by McLaughlin and Scott. ${ }^{2}$ This device, as well as providing a convenient mathematical model because of periodic boundary conditions, has in recent years begun to attract research interest in its own right. ${ }^{3,4}$

The paper is structured as follows. In Sec. II we describe the mathematical model of the circular junction. Details of the numerical techniques employed are presented in Sec. III. In Sec. VI we study the behavior of the oscillator under the influence of a sinusoidal driving term in the bias current, which models external microwave irradiation. Section V contains calculations of the linewidth under the influence of Gaussian white noise, which models internal thermal noise in the junction. In Sec. VI we compare our results with existing experimental observations. In all of the sections we are focusing on a configuration with a single propagating fluxon, which corresponds to the first zero-field step in the current-voltage characteristic of the oscillator.

\section{MATHEMATICAL MODEL}

As a model for the Josephson tunnel junction of overlap geometry we use the perturbed sine-Gordon equation, ${ }^{5}$

$$
\varphi_{x x}-\varphi_{t t}-\sin \varphi=\alpha \varphi_{t}+\gamma+\eta(x, t)
$$

Here $\varphi$ is the quantum phase difference between the two superconducting layers in the junction. Space and time are normalized to the Josephson penetration length $\lambda_{J}=\left(\Phi_{0} / 2 \pi j_{0} L_{p}\right)^{1 / 2}$, and the inverse of the plasma frequency $\omega_{p}=\left(2 \pi j_{0} / \Phi_{0} C\right)^{1 / 2}$, respectively, where $\Phi_{0}$ is the magnetic flux quantum given by $\Phi_{0}=h / 2 e=2.064$ $\times 10^{-15} \mathrm{~Wb} . L_{p}$ and $C$ are the inductance and the capacitance per unit length of the junction. The first of the perturbation terms on the right-hand side of Eq. (2.1) represents the loss due to tunneling of normal electrons, in normalized units $\alpha=G / \omega_{p} C$, where $G^{-1}$ is an effective normal resistance per unit length. The second term is the normalized bias current $\gamma$ measured in units of $j_{0}$ the maximum Josephson current per unit length. In this paper we include a third term $\eta(x, t)$ representing either an externally applied sinusoidal driving term connected to the bias, or an internal thermal noise term connected to the loss. In this second case we assume a distributed Gaussian white noise with zero mean value.

The normalized length of the Josephson junction $l=L / \lambda_{J}$ is assumed to be large compared with unity and the normalized width $w=W / \lambda_{J}$ small compared with unity, allowing us to use a $1+1$ dimensional model. ${ }^{6} \mathrm{Be}-$ cause the aim of this investigation is to isolate the influence of the term $\eta(x, t)$ on the solution to Eq. (2.1) we avoid phenomena connected with collision with junction boundaries by considering a long annular junction. Therefore, we demand spatial periodicity with period $l$ in the two physical quantities, the voltage drop across the junction:

$$
V=\frac{\Phi_{0} \omega_{p}}{2 \pi} \varphi_{t}
$$

and the current along the junction,

$$
I=-j_{0} \lambda_{J} \varphi_{x},
$$

i.e., boundary conditions

$$
\begin{aligned}
& \varphi_{t}(0, t)=\varphi_{t}(l, t), \\
& \varphi_{x}(0, t)=\varphi_{x}(l, t) .
\end{aligned}
$$


The fluxon traveling wave solution to the unperturbed version of Eq. (2.1) is given by ${ }^{7}$

$$
\varphi=2 \sin ^{-1}[\operatorname{cn}(\xi, k)],
$$

with $\xi=(x-u t) /\left[k\left(1-u^{2}\right)^{1 / 2}\right]$. Here $u$ is the velocity of the wave and $k$ is the modulus in the Jacobian elliptic function. $^{8}$ Spatial periodicity requires $l /\left(1-u^{2}\right)^{1 / 2}$ $=2 n k K(k)$, where $n$ is the winding number, i.e., the number of fluxons minus the number of antifluxons, and $K(k)$ is the complete elliptic integral of the first kind. In Ref. 9 it is shown by Hamiltonian perturbation theory ${ }^{2}$ that the steady-state fluxon velocity dependence on the loss and bias parameters is

$$
u=1 /\left(1+\left(4 \alpha^{\prime} / \pi \gamma\right)^{2}\right)^{1 / 2},
$$

with $\alpha^{\prime}=\alpha E(k) / k$, where $E(k)$ is the complete elliptic integral of the second kind. For $l \geq 8$ (assuming $n=1$ ) Eq. (2.5) reduces to the kink for the infinite line $\varphi=4 \tan ^{-1}\left(e^{\xi}\right)$ with $\xi=(x-u t) /\left(1-u^{2}\right)^{1 / 2}$, and the velocity given by $u=1 /\left[1+(4 \alpha / \pi \gamma)^{2}\right]^{1 / 2}$. In the numerical simulations we have used $l=8,12.8,20$, and $n=1$.

\section{NUMERICAL TECHNIQUES}

The very narrow linewidth of the radiation emitted from a Josephson-junction oscillator (less than $1 \mathrm{kHz}$ at $10 \mathrm{GHz})^{10}$ suggests that a relative numerical accuracy of at least $10^{-7}$ is essential. We solve Eq. (2.1) numerically by using a pseudospectral method. ${ }^{11}$ This method, a Fourier transform treatment in space together with a leapfrog scheme in time, has the advantage of simplicity and high-order accuracy in the approximations to the space derivatives. Expansion of the fluxon wave into truncated series of sines and cosines demands periodicity not only in $\varphi_{x}$ and $\varphi_{t}$ but also in $\varphi$ itself. Observing that the fluxon is a localized kink connecting two ground states separated by $2 \pi$ we introduce a new periodic function $\varphi-2 \pi x / l$ whose Fourier representation we denote $\Phi^{p}(t)$ with the superscript $p=0, \pm 1, \ldots, \pm p_{\max }$.

Transforming Eq. (2.1) into the following set of ordinary nonlinear coupled differential equations:

$$
\begin{aligned}
& -k_{p}^{2} \Phi^{p}(t)-\Phi_{t t}^{p}(t)-F^{p}\{\sin \varphi\} \\
& =\alpha \Phi_{t}^{p}(t)+l \gamma \delta_{p, 0}+N^{p}(t), \\
& k_{p}=2 \pi p / l, \quad p=0, \pm 1, \ldots, \pm p_{\max }
\end{aligned}
$$

in which $F^{p}$ and $N^{p}$ are the Fourier components of $\sin \varphi$ and $\eta$, respectively, and $\delta_{p, 0}$ denotes the Kronecker symbol, and using second-order central differences to approximate the time derivatives we get an explicit scheme for the time evolution of the Fourier components

$$
\begin{aligned}
\Phi_{j+1}^{0}=[ & 2 \Phi_{j}^{0}-(1-\alpha \Delta t / 2) \Phi_{j-1}^{0} \\
& \left.-\Delta t^{2}\left(S_{j}^{0}+l \gamma+N_{j}^{0}\right)\right] /(1+\alpha \Delta t / 2), \\
\Phi_{j+1}^{p}=[ & \left(2-\Delta t^{2} k_{p}^{2}\right) \Phi_{j}^{p}-(1-\alpha \Delta t / 2) \Phi_{j-1}^{p} \\
& \left.-\Delta t^{2}\left(S_{j}^{p}+N_{j}^{p}\right)\right] /(1+\alpha \Delta t / 2), \quad|p|>0,
\end{aligned}
$$

where $S_{j}^{p}$ equals $F^{p}\{\sin \varphi\}$ at time $j \Delta t$, calculated each time step by transforming $\Phi_{j+1}^{p}$ back to $x$ space, calculat-

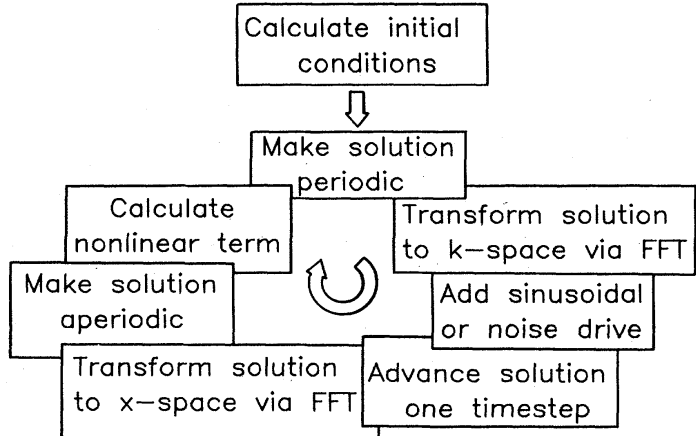

FIG. 1. Schematic diagram of numerical simulation procedure.

ing $\sin \varphi$ and then transforming again to $k$ space as indicated schematically in Fig. 1.

Figure 2 shows the computed $\varphi_{x}$ as a function of time at an arbitrary point on the junction. This signal consists of an almost-periodic sequence of pulses. In fact, it is the deviation from perfect periodicity that gives a nonzero linewidth of the radiation. Since the deviation is small it is necessary to devise a very accurate method for determining the revolution periods $T_{n}$ for the circulating fluxon. We do this by calculating $T_{n}$ as the time for the mean value of the phase over $x$ to change by $2 \pi$. The fundamental frequency of the signal then becomes $f_{0}=1 /\left\langle T_{n}\right\rangle$, where brackets denote an average value. We take the power spectrum of the signal near $f_{0}$ to be the distribution of the computed values of $1 / T_{n}$.

Figure 3 shows the calculated $T_{n}$ 's in a computer experiment with the driving term $\eta=0$ in Eq. (2.1). As can be seen from Fig. 3, the relative accuracy $\Delta T /\left\langle T_{n}\right\rangle \lesssim 10^{-8}$. In fact, examination of the numerical output shows that it is approximately $7 \times 10^{-9}$. The long transient arises from the fact that the initial conditions given by

$$
\begin{aligned}
& \varphi(x, 0)=f(x, 0)-\sin ^{-1}(\gamma), \\
& \varphi(x,-\Delta t)=f(x,-\Delta t)-\sin ^{-1}(\gamma),
\end{aligned}
$$

where $f(x, t)$ is the fluxon traveling wave solution to the unperturbed sine-Gordon equation as given by Eq. (2.5)

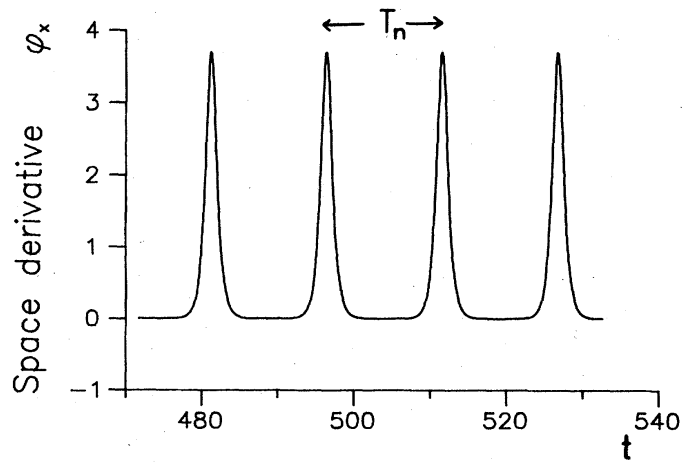

FIG. 2. Time dependence of the space derivative of the fluxon wave form, showing the $n$th period of revolution $T_{n}$ for $\alpha=0.01, \gamma=0.02, \eta=0$, and $l=12.8$. 


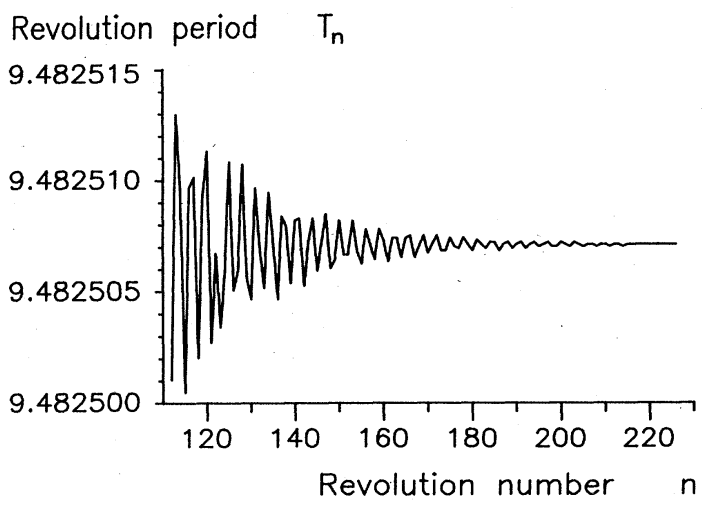

FIG. 3. Revolution period $T_{n}$ as a function of revolution number $n$ for $\alpha=0.01, \gamma=0.02, \eta=0$, and $l=8$ showing high level of computational accuracy achieved.

and $\sin ^{-1}(\gamma)$ is the ground state, are not exactly equal to the final propagating configuration.

We note at this point that the accuracy of the results was checked by doubling $p_{\max }$ in Eq. (3.1b), in order to ensure that no spurious Fourier modes due to the discretization in $x$ space are produced, and halving $\Delta t$ in Eqs. (3.2). The values used for $p_{\max }$ ranged from 64 to 256 and those for $\Delta t$ from 0.075 to 0.0025 , depending on the parameters $l$ and $\gamma$.

The computer program was implemented on an IBM 3033 in double precision (approximately 16 significant digits) and on a CRAY-1 vector processor in single precision (approximately 15 significant digits) using optimizing FORTRAN compilers. In the former case we have used the IMSL-routine FFT2C for fast Fourier transform. ${ }^{12}$ In the latter case, by making full use of vectorization of the computer code and the CRAY routines for Fourier transform and vector copying CFFT2 (Ref. 13) and CCOPY (Ref. 14) we gained a speed-up factor in computing time of 22. Each long simulation requires typically $5 \times 10^{5}$ time steps on a 512 -point spatial lattice and uses approximately $10 \mathrm{~min}$ of CPU time on the CRAY-1-S as opposed to approximately $4 \mathrm{~h}$ on a scalar machine.

Finally, we have compared the steady-state fluxon velocity, given by $u=l /\left\langle T_{n}\right\rangle$, with the predicted value

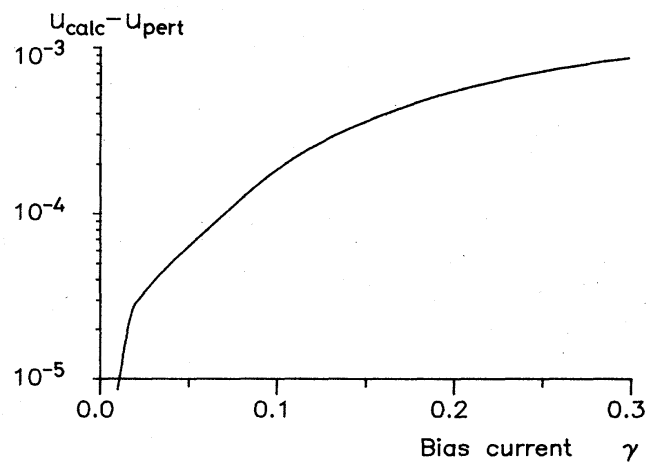

FIG. 4. Difference between average propagation velocity as computed numerically $u_{\text {calc }}$ and calculated from perturbation theory $u_{\text {pert }}$ from Eq. (2.6) as a function of the bias for $\alpha=0.01$, $\eta=0$, and $l=8$. from Hamiltonian perturbation theory, Eq. (2.6). The result is seen in Fig. 4. The deviation for large bias values is expected because the perturbation theory is only valid for small $\gamma$ values.

\section{SINUSOIDAL DRIVING TERM}

In this section we investigate the behavior of the fluxon velocity when the driving term is given by

$$
\eta(x, t)=\eta(t)=\eta_{0} \sin (\Omega t),
$$

as a function of the driving frequency $\Omega$. This might be considered as a model of microwave irradiation of the junction. Using the definition of the normalized momentum

$$
p(t)=-\frac{1}{8} \int_{0}^{l} \varphi_{x} \varphi_{t} d x,
$$

and separating the phase into a kink part and a background $\operatorname{part}^{15} \varphi(x, t)=\varphi^{k}(x, t)+\varphi^{\infty}(t)$, and assuming that the length of the junction is large, allowing expressions for the infinite junction to be used, we get the following equation for the momentum $p^{k}$ of the kink,
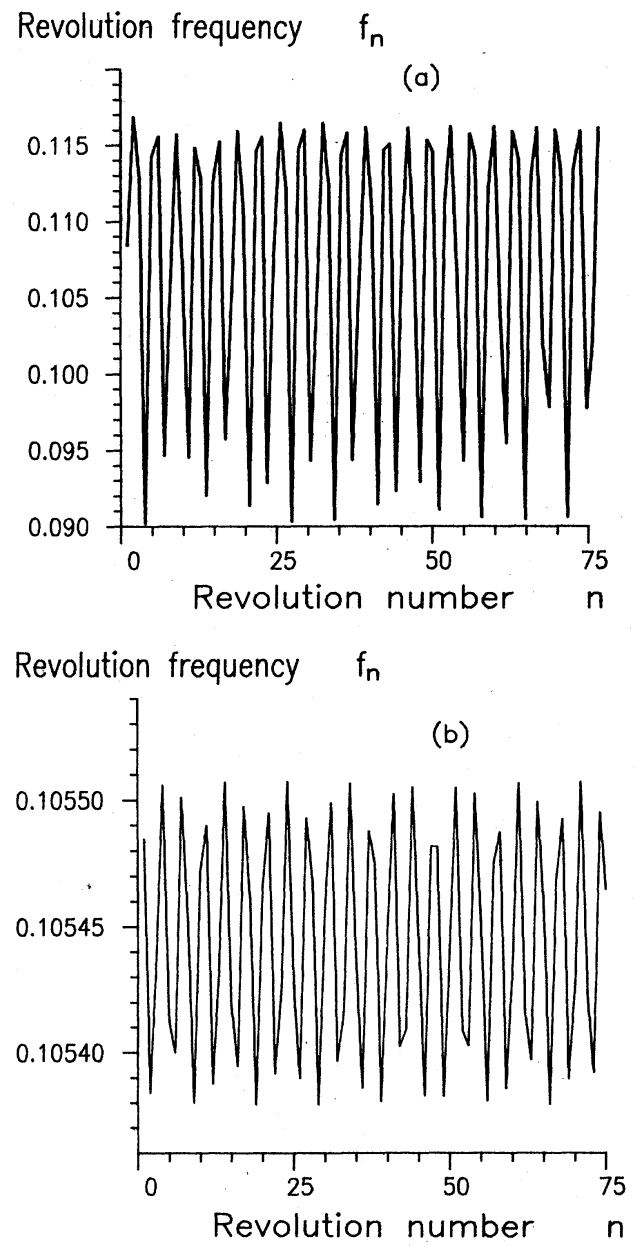

FIG. 5. Revolution frequency $f_{n}$ as a function of revolution number $n$ for sinusoidal drive, $\eta(t)=\eta_{0} \sin (\Omega t)$, with $\alpha=0.01$, $\gamma=0.02, \Omega=0.86, \eta_{0}=0.01$, and $l=8$. (a) Numerical simulation. (b) Kink model. 


$$
\frac{d p^{k}}{d t}+\alpha p^{k}=\frac{\pi}{4}\left[\gamma+\eta_{0} \sin (\Omega t)+\alpha \frac{d \varphi^{\infty}}{d t}+\frac{d^{2} \varphi^{\infty}}{d t^{2}}\right)
$$

Thus, the background motion becomes an effective driving term for the kink part. From Eq. (2.1) we derive the linearized equation for $\hat{\varphi}^{\infty}=\varphi^{\infty}+\sin ^{-1}(\gamma)$, assuming that $\hat{\varphi}{ }^{\infty} \ll 1$,

$$
\frac{d^{2} \hat{\varphi}^{\infty}}{d t^{2}}+\alpha \frac{d \hat{\varphi}^{\infty}}{d t}+\left(1-\gamma^{2}\right)^{1 / 2} \hat{\varphi}^{\infty}=-\eta_{0} \sin (\Omega t) .
$$

Combining Eqs. (4.3) and (4.4) we obtain for the kink momentum

$$
\begin{aligned}
p^{k}(t)=\frac{\pi}{4}[ & \frac{\gamma}{\alpha}+\frac{\eta_{0}}{\left(\alpha^{2}+\Omega^{2}\right)^{1 / 2}} \sin \left(\Omega t-\theta_{1}\right) \\
& \left.-\frac{\eta_{0} \Omega}{\left\{\left[\left(1-\gamma^{2}\right)^{1 / 2}-\Omega^{2}\right]^{2}+\alpha^{2} \Omega^{2}\right\}^{1 / 2}} \cos \left(\Omega t-\theta_{2}\right)\right],
\end{aligned}
$$
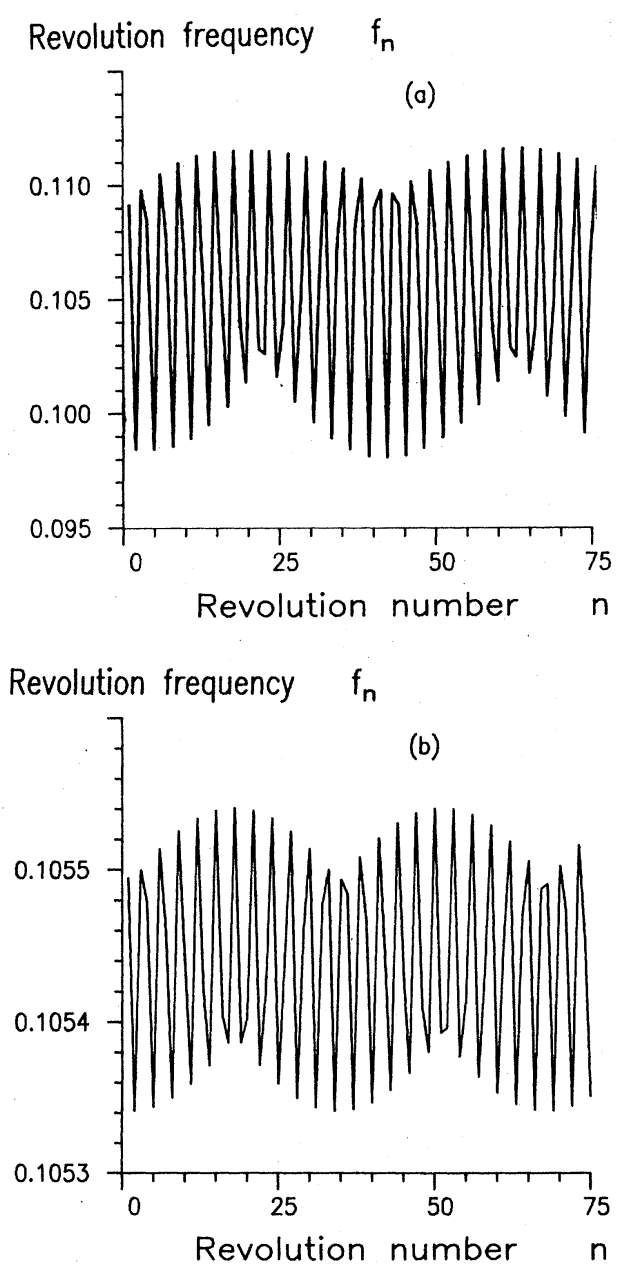

FIG. 6. Revolution frequency $f_{n}$ as a function of revolution number $n$ for sinusoidal drive, $\eta(t)=\eta_{0} \sin (\Omega t)$, with $\alpha=0.01$, $\gamma=0.02, \Omega=0.89, \eta_{0}=0.01$, and $l=8$. (a) Numerical simulation. (b) Kink model.

$$
\theta_{1}=\tan ^{-1}(\Omega / \alpha)
$$

and

$$
\theta_{2}=\tan ^{-1}\left\{\alpha \Omega /\left[\left(1-\gamma^{2}\right)^{1 / 2}-\Omega^{2}\right]\right\} .
$$

The instantaneous kink velocity is then calculated from $p^{k}=u /\left(1-u^{2}\right)^{1 / 2}$. In order to compare this approximate theoretical description with the numerical result we calculate the $n$th period $T_{n}$ according to the formula

$$
\int_{t_{n-1}}^{t_{n-1}+T_{n}} u d t=l
$$

with

$$
u=p^{k} /\left[1+\left(p^{k}\right)^{2}\right]^{1 / 2}
$$

Figures 5-7 show a comparison of the results from this linearized model and from numerical simulations of Eq. (2.1) with $\Omega=0.86,0.89$, and 1.10 , respectively. In all cases it is seen that the kink model is able to reproduce the fluctuations in the revolution frequency $f_{n}=1 / T_{n}$ in great detail.

As a measure of the amplitude of the frequency fluc-
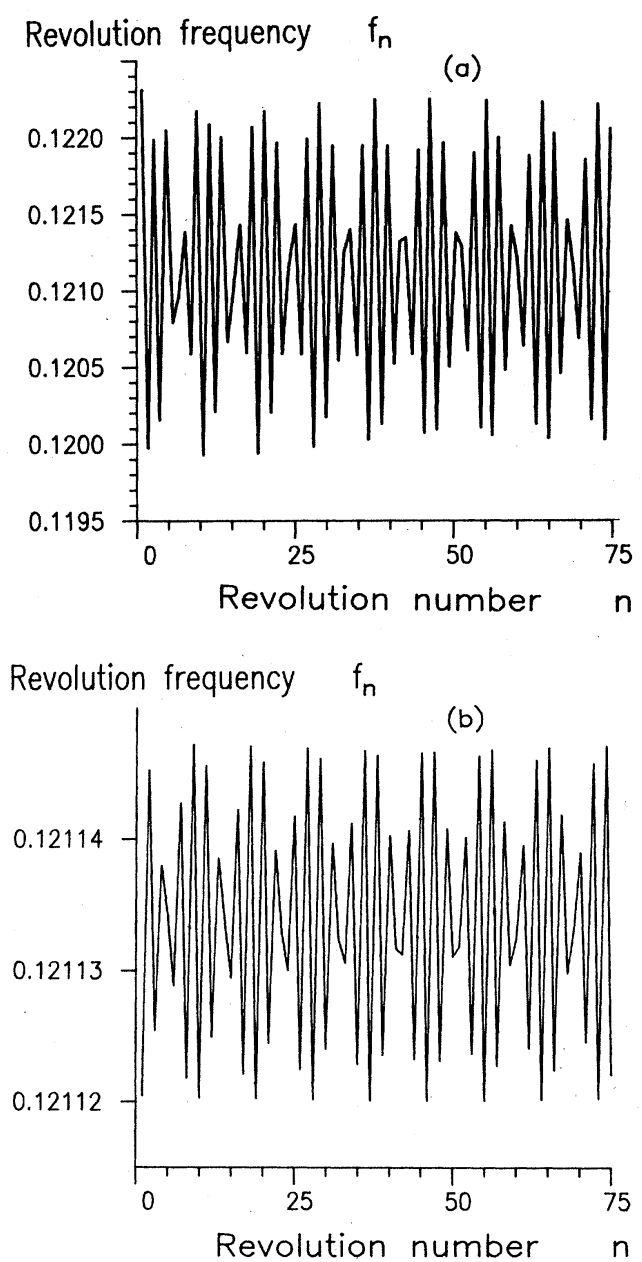

FIG. 7. Revolution frequency $f_{n}$ as a function of revolution number $n$ for sinusoidal drive, $\eta(t)=\eta_{0} \sin (\Omega t)$, with $\alpha=0.01$, $\gamma=0.05, \Omega=1.10, \eta_{0}=0.01$, and $l=8$. (a) Numerical simulation. (b) Kink model. 


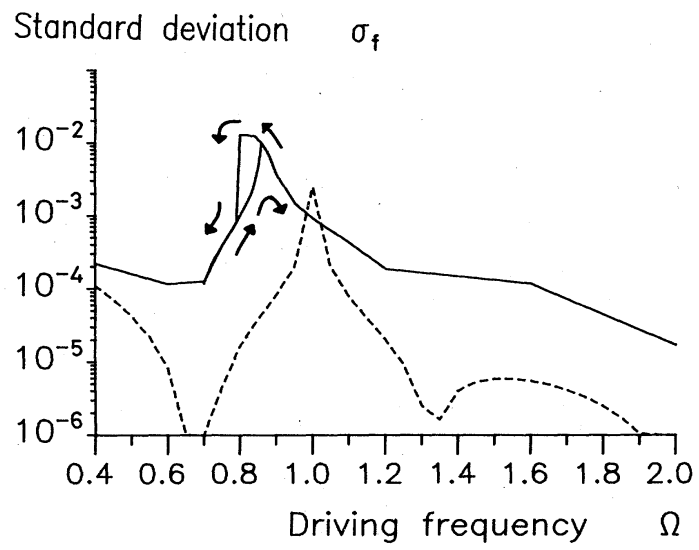

FIG. 8. Standard deviation of revolution frequency $\sigma_{f}$ as a function of driving frequency $\Omega$. Solid curve, numerical simulation; dashed curve, kink model; parameters, $\alpha=0.01, \gamma=0.02$, $\eta_{0}=0.01$, and $l=8$.

tuation, which is essentially the linewidth of the oscillator, we have calculated the standard deviation of the revolution frequency $\sigma_{f}=\left[\left\langle\left(f_{n}-\left\langle f_{n}\right\rangle\right)^{2}\right\rangle\right]^{1 / 2}$ for values of the cyclic driving frequency $\Omega$ between 0.4 and 2.0 .

The full curve in Fig. 8 shows the results from the numerical simulation and the dashed curve those from the kink model. The kink model predicts a resonance just below the plasma frequency $\Omega=1$, whereas the numerical simulation yields this peak at a somewhat lower frequency. Moreover, the numerical results exhibit a hysteresis not seen in those of the kink model and a difference in scale. The discrepancy in resonance frequency and hysteresis behavior is attributable to the fact that we have used a linearized kink model. Presumably, the use of a higher-order expansion in Eq. (4.4) would yield a behavior analogous to that of a soft nonlinear spring ${ }^{16}$ thus reducing these discrepancies. It is not clear, however, to what extent the difference in scale would be resolved by such a refinement.

\section{GAUSSIAN WHITE NOISE}

The term $\eta(x, t)$ in Eq. (2.1) is here considered to be Gaussian white noise with zero mean $\langle\eta(x, t)\rangle=0$ and autocorrelation function

$$
R_{\eta}(\zeta, \tau)=\langle\eta(x, t) \eta(x+\zeta, t+\tau)\rangle=\sigma_{\eta}^{2} \delta(\zeta) \delta(\tau) .
$$

The variance of the noise $\sigma_{\eta}^{2}$ is connected with the loss $\alpha$ and the absolute temperature $T$ through $^{17}$

$$
\sigma_{\eta}^{2}=4 \pi \alpha k T / \Phi_{0} j_{0} \lambda_{J},
$$

where $k$ is the Boltzmann constant.

In the vectorized algorithm we find it convenient to introduce the noise term in $p-t$ space, $N^{p}(t)$, as

$$
N^{p}(t)=F^{-1}\left\{\sigma_{\eta} \exp \left(i\left(\theta_{p}+\theta_{\omega}\right)\right)\right\},
$$

where $F^{-1}$ denotes the Fourier transform from $\omega$ to $t$ space, and $\theta_{p}$ and $\theta_{\omega}$ are stochastic variables uniformly distributed between 0 and $2 \pi$, with an upper limit in $p$ and $\omega$ of $p_{\max }=1 / 2 \Delta x$ and $\omega_{\max }=\pi / \Delta t, \Delta x$ and $\Delta t$ being the resolution in space and time, respectively. Standard
Hamiltonian perturbation theory for the fluctuations $\Delta u$ in the fluxon velocity leads to the power spectrum for $\Delta u,{ }^{10}$

$$
S_{\Delta u}(\omega)=\frac{1}{8} \sigma_{\eta}^{2}\left(1-u_{0}^{2}\right)^{5 / 2} \frac{1}{\omega^{2}+\alpha^{2}},
$$

with the average velocity $u_{0}$ given by Eq. (2.6). By a Fourier transform of Eq. (5.4) we obtain the autocorrelation function for $\Delta u$ as an exponential

$$
R_{\Delta u}(\tau)=\frac{\sigma_{\eta}^{2}\left(1-u_{0}^{2}\right)^{5 / 2}}{16 \alpha} e^{-\alpha|\tau|}
$$

Thus $\Delta u(t)$ is a normal process with zero mean and standard deviation ${ }^{18}$

$$
\sigma_{\Delta u}=\frac{\sigma_{\eta}\left(1-u_{0}^{2}\right)^{5 / 4}}{4 \alpha^{1 / 2}} .
$$

Defining the period of a fluxon revolution according to Eq. (4.6) we calculate the average frequency fluctuation as the average of the instantaneous frequency fluctuation $\Delta u / l$ over one average period of revolution

$$
\Delta f=\frac{1}{\langle T\rangle} \int_{t}^{t+\langle T\rangle} \Delta u / l d t
$$

From Eq. (5.7) it follows that $\Delta f$ has a normal distribution with zero mean and the standard deviation, ${ }^{19}$

$\sigma_{\Delta f}=\frac{1}{l} \sigma_{\Delta u}\left[\frac{2 u_{0}}{\alpha l}\left[1-u_{0} \frac{1-\exp \left(-\alpha l / u_{0}\right)}{\alpha l}\right]\right]^{1 / 2}$.

A numerical simulation with $\sigma_{\eta}=8.8 \times 10^{-4}$ is seen in Fig. 9 showing a typical frequency distribution of $\Delta f$ about the fundamental frequency $f_{0}=u_{0} / l$. The connection between the standard deviation and the half-power linewidth is

$$
\Delta f_{1 / 2}=\sqrt{8 \ln 2} \sigma_{\Delta f}
$$

when $\Delta f$ is normal distributed.

Figures 10 and 11 show a comparison of the standard deviation predicted by this model Eq. (5.8) and the results

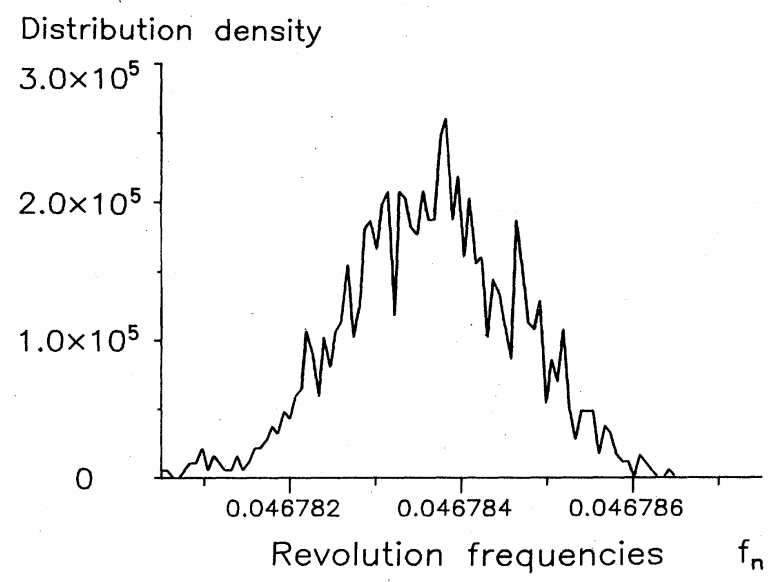

FIG. 9. Distribution of revolution frequency $f_{n}$. Numerical simulation with Gaussian noise drive: $\alpha=0.01, \gamma=0.034$, $\sigma_{\eta}=8.8 \times 10^{-4}$, and $l=20$. 

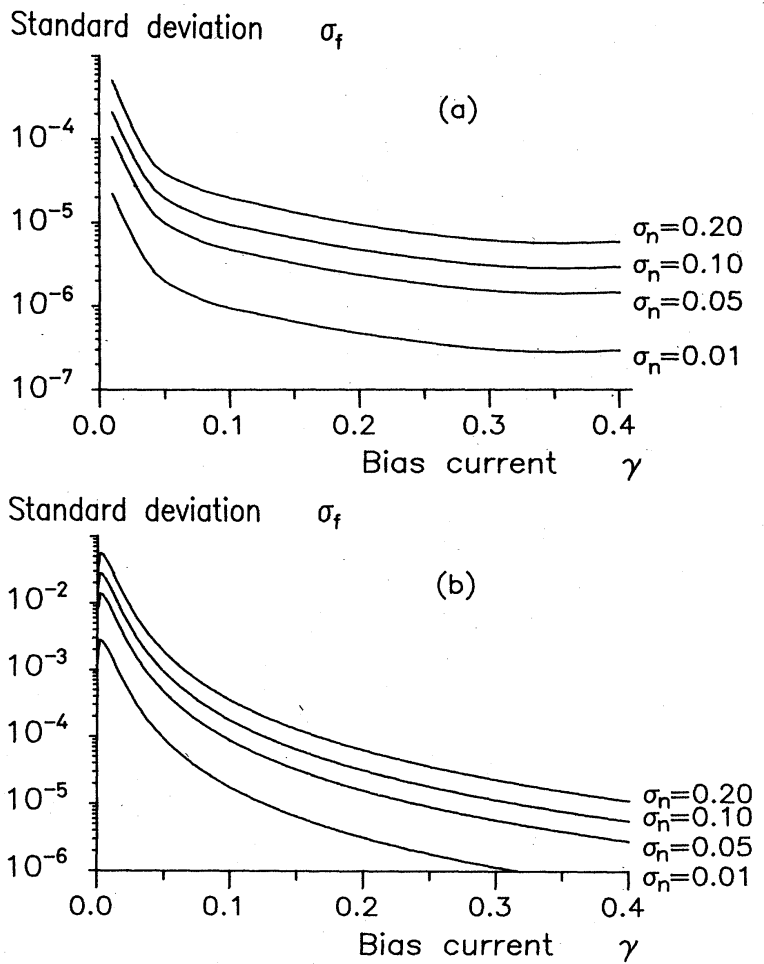

FIG. 10. Standard deviation of revolution frequency $\sigma_{f}$ for white Gaussian noise drive as a function of bias current $\gamma$, for $\alpha=0.01, l=8$, and $\sigma_{\eta}=0.01,0.05,0.10$, and 0.20 . (a) Numerical simulation. (b) Hamiltonian perturbation theory.
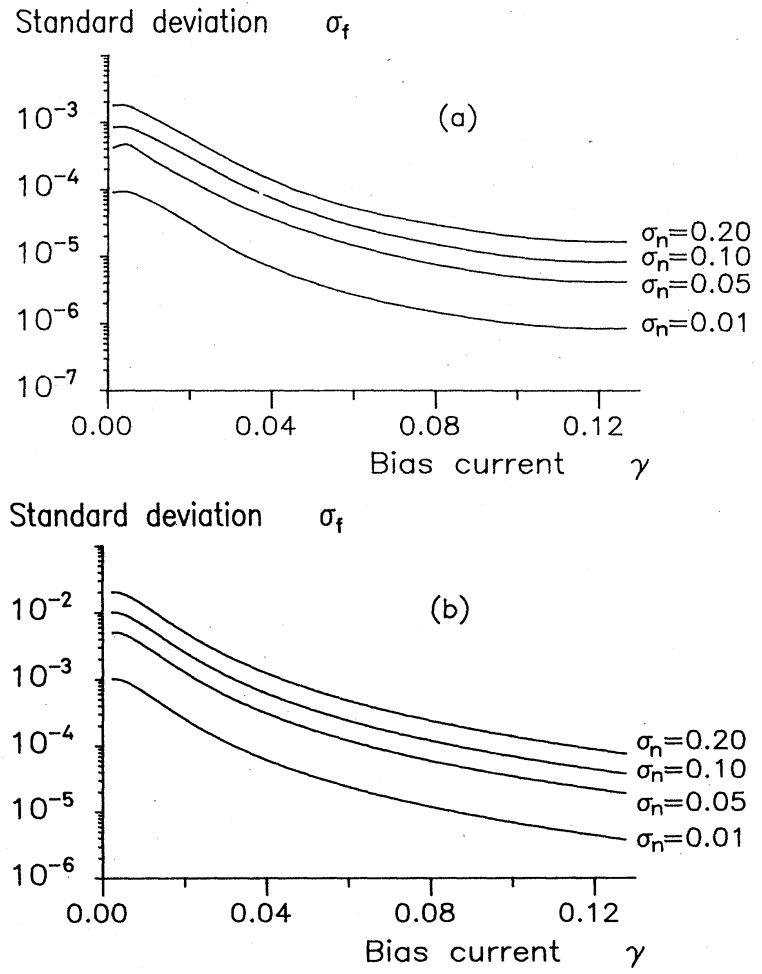

FIG. 11. Standard deviation of the revolution frequency $\sigma_{f}$ for white Gaussian noise drive as a function of bias current $\gamma$, for $\alpha=0.01, l=20$, and $\sigma_{\eta}=0.01,0.05,0.10$, and 0.20 . (a) $\mathrm{Nu}$ merical simulation. (b) Hamiltonian perturbation theory. from the numerical simulations for the lengths $l=8$ and $l=20$, respectively. As can be seen, the model is able to predict the right qualitative dependence on the length, the noise amplitude, and the bias, but the model predicts an overall standard deviation that is about a factor of 10 too large. The reason for this discrepancy is at present not known.

In closing, we note that for $\gamma$ values near 0.3 it was found necessary to augment the time resolution (by reducing $\Delta t$ ) to avoid spurious peaks in Fig. 10(a). The existence of such spurious peaks might be an indicator of the onset of chaotic behavior at nearby points in parameter space. In fact, parameter values $\gamma=0.3$ with $\alpha=0.01$ lead to chaotic creation of fluxon-antifluxon pairs in the study reported by Eilbeck et $a .^{20}$

\section{COMPARISON WITH EXPERIMENTS}

The rapidly decreasing linewidth with increasing bias shown in Figs. 10 and 11 is in qualitative agreement with the experimental observations of Fig. 1 in Ref. 10.

To compare quantitatively the calculated results with these experiments we use in Eq. (5.2) data reported by Scott et al. ${ }^{5}$ For the junction No. N25L, assuming a temperature of $4 \mathrm{~K}$, Eq. (5.2) gives $\sigma_{\eta}=0.0052$. Noting from Fig. 9(a) that $\sigma_{f}$ scales linearly with $\sigma_{\eta}$, we calculate from Eq. (5.9) a normalized half-power linewidth $\Delta f_{1 / 2}=5.5 \times 10^{-7}$ at $\gamma=0.2$. Taking as the normalized resonance frequency $f_{0}=u_{0} / l \simeq 0.125$ we calculate a relative linewidth $\Delta f_{1 / 2} / f_{0}=4.4 \times 10^{-6}$. The physical resonance frequency for junction No. N25L was $2.3 \mathrm{GHz}{ }^{5}$ This yields a physical linewidth of $10 \mathrm{kHz}$. Comparing with the experimental results shown in Fig. 1 of Ref. 10 and noting that $\gamma=0.2$ corresponds to a bias point near the bottom of the zero-field step, we find excellent agreement. The same calculations for junction No. N53C, ${ }^{5}$ again for $T=4 \mathrm{~K}$ and $\gamma=0.2$, yield $\Delta f_{1 / 2} / f_{0}$ $=2.3 \times 10^{-6}$. The physical resonance frequency for junction No. N53C was $8.3 \mathrm{GHz}$, which leads to a physical linewidth of $18 \mathrm{kHz}$, once again in excellent agreement with experimental results.

\section{CONCLUSIONS}

Computational studies of the linewidth of the radiation emitted by Josephson junctions require extremely high resolution. For this reason we developed a pseudospectral method for solving the nonlinear dynamical equation describing a circular Josephson junction oscillator. Because the algorithm makes heavy use of fast Fourier transforms it was implemented on a CRAY-1 vector processor. Driving terms corresponding to physically realistic situations, i.e., sinusoidal microwave irradiation and internal thermal noise, were considered. In the second case the computational results were compared with experimental results reported in the literature, and excellent qualitative and quantitative agreement was found. In addition, in both cases we have compared the computational results with approximate analytic results based on perturbation theory. Here the agreement was qualitatively good, but quantitative discrepancies were found, indicating a need for further development of perturbation theory. 


\section{ACKNOWLEDGMENTS}

It is our pleasure to thank Alwyn C. Scott for proposing this work and for his continued interest in it. We acknowledge Niels F. Pedersen for helpful discussions on modeling of thermal noise in Josephson junctions and Mogens Samuelsen for pointing out the significance of
Ref. 15 in the context of the present work. The financial support of the Danish Council for Scientific and Industrial Research and by the European Research Office of the United States Army (through Contract No. DAJA-37-82C-0057) is gratefully acknowledged. R.D.P. thanks the Technical University of Denmark for providing support for part of the period during which this work was performed.
*Permanent address: Dipartimento di Fisica, Università di Salerno, 84100 Salerno, Italy.

${ }^{1}$ N. F. Pedersen, in Advances in Superconductivity, NATO Advanced Study Institute Series, B100, edited by B. Deaver and J. Ruvalds (Plenum, New York, 1982), p. 149.

${ }^{2}$ D. W. McLaughlin and A. C. Scott, Phys. Rev. A 18, 1652 (1978).

${ }^{3}$ A. Davidson and N. F. Pedersen, Appl. Phys. Lett. 44, 465 (1984).

${ }^{4}$ B. Dueholm, A. Davidson, C. C. Tsuei, M. J. Brady, K. H. Brown, A. C. Callegari, M. M. Chen, J. H. Greiner, H. C. Jones, K. K. Kim, A. W. Kleinsasser, H. A. Notarys, G. Proto, R. H. Wang, and T. Yogi, Proceedings of the 17th International Conference on Low-Temperature Physics, Conference LT 17, edited by U. Eckern, A. Schmid, W. Weber, and H. Wühl (North-Holland, Amsterdam, 1984), Part I, p. 691.

${ }^{5}$ A. C. Scott, F. Y. F. Chu, and S. A. Reible, J. Appl. Phys. 47, 3272 (1976).

6J. C. Eilbeck, P. S. Lomdahl, O. H. Olsen, and M. R. Samuelsen, J. Appl. Phys. (to be published).

${ }^{7}$ R. D. Parmentier, in Solitons in Action, edited by K. Lonngren and A. C. Scott (Academic, New York, 1978), p. 173.

${ }^{8}$ P. F. Byrd and M. D. Friedman, Handbook of Elliptic Integrals for Engineers and Physicists (Springer, Berlin, 1954).

${ }^{9}$ F. If, M. P. Soerensen, and P. L. Christiansen, Phys. Lett. 100A, 68 (1984).
${ }^{10}$ E. Joergensen, V. P. Koshelets, R. Monaco, J. Mygind, M. R. Samuelsen, and M. Salerno, Phys. Rev. Lett. 49, 1093 (1982).

${ }^{11}$ D. Gottlieb and S. A. Orszag, Numerical Analysis of Spectral Methods (Society for Industrial and Applied Mathematics, Philadelphia, 1977).

12IMSL, Inc. (Houston, Texas) International Mathematical and Statistical Library (9th Ed.) routine FFT2C, 1982 (unpublished).

${ }^{13}$ CRAY-1 Computer Systems, Library Reference Manual SR0014 (Cray Research, Mendota Heights, 1982), pp. 4-49; see also CRAY Computer Systems Technical Note, Complex Fast Fourier Transform Binary Radix Subroutine (CFFT2) SN0203 (Cray Research, Mendota Heights, 1983).

${ }^{14}$ CRAY-1 Computer Systems, Library Reference Manual SR0014 (Cray Research, Mendota Heights, 1982), pp. 4-9.

${ }^{15}$ O. A. Levring, M. R. Samuelsen, and O. H. Olsen, Physica 11D, 349 (1984).

${ }^{16}$ A. H. Nayfeh and D. T. Mook, Nonlinear Oscillations (Wiley, New York, 1979), p. 161.

${ }^{17}$ M. Büttiker and R. Landauer, Phys. Rev. A 23, 1397 (1981).

${ }^{18}$ A. Papoulis, Probability, Random Variables, and Stochastic Processes (McGraw-Hill, New York, 1965), p. 519.

${ }^{19}$ A. Papoulis, Probability, Random Variables, and Stochastic Processes (Ref. 18), p. 324.

20J. C. Eilbeck, P. S. Lomdahl, and A. C. Newell, Phys. Lett. 87A, 1 (1981). 